This is the peer reviewed version of the following article: Blake, M., Bowes, A., Gill, V., Husain, F. and Mir, G. (2017), A collaborative exploration of the reasons for lower satisfaction with services among Bangladeshi and

Pakistani social care users. Health Soc Care Community, 25: 1090-1099, which has been published in final form at https://doi.org/10.1111/hsc.12411. This article may be used for non-commercial purposes in accordance With Wiley Terms and Conditions for self-archiving. 


\title{
A collaborative exploration of the reasons for lower satisfaction with services among Bangladeshi and Pakistani ${ }^{1}$ social care users
}

\author{
AUTHORS: \\ Ghazala Mir, PhD

\section{Correspondence} \\ Fatima Husain, $\mathrm{PhD}$ \\ Research Director \\ NatCen Social Research \\ 35 Northampton Square \\ London EC1V OAH \\ England \\ E-mail: fatima.husain@natcen.ac.uk
}

Margaret Blake, PhD, Prof Alison Bowes, PhD, Valdeep Gill, MSc, Fatima Husain, PhD,

NatCen Social Research, 35 Northampton Square, London, EC1V 0AX, England, The School of Applied Social Science, University of Stirling, Stirling, FK9 4LA, Scotland, Leeds Institute of Health Sciences, Charles Thackrah Building, University of Leeds, 101 Clarendon Road, Leeds, LS2 9LJ, England, and Ipsos MORI, 3 Thomas More Square, London EW1 1YW.

Accepted for publication in Health and Social Care in the Community published by Wiley-Blackwell.

\section{Source of funding}

This study was funded by the National Institute for Health Research, School for Social Care Research (NIHR SSCR) under Grant T976/T11-017/NCMB.

The views expressed are those of the authors and not necessarily those of the NIHR SSCR or the DH, NIHR or NHS.

\footnotetext{
${ }^{1}$ It is important to note that 'Bangladeshi' and 'Pakistani' in the context of this research mean individuals of Bangladeshi and Pakistani origin who are resident in the UK and may or may not have British nationality.
} 


\section{Abstract}

This study explored underlying reasons for the expression of dissatisfaction with services among Bangladeshi and Pakistani social care users in England and investigated, using a collaborative approach, how these could be addressed.

In-depth interviews were conducted in Birmingham, Leeds and London during 2012 13 with 63 Bangladeshi, Pakistani and white British service users and 24 social care managers, social workers and care workers. A further 34 cognitive interviews were conducted within the same study. Following data analysis, three collaborative workshops involving service users and providers were held to validate the findings and to draw out policy and practice recommendations.

Analysis of the cognitive interviews showed that higher dissatisfaction amongst Bangladeshi and Pakistani service users reported in social care surveys was not due to questionnaire design. Instead in-depth interviews showed that dissatisfaction across all three groups was expressed along the social care journey, including accessing care, communication with social workers and, the nature of care received. Whilst many issues were common to all three groups, cultural differences also emerged as affecting experiences of social care. These included misunderstandings about family roles in care; gender issues, especially relating to women; language and communication barriers, alongside the need for a more nuanced approach to ethnic 'matching'; and continuing limited cultural understanding among care workers. The collaborative workshops identified practical actions that could address some of the issues identified. These covered raising awareness of services within communities; improving support for informal carers; service user input to assessments; consistent and on-going sharing of information; improving access; and more efforts to diversify and appropriately train the social care workforce.

In conclusion, the paper presents reality of dissatisfaction among these groups and argues for more action involving communities and service providers to address these persistent issues collaboratively.

\section{Keywords}

Adult social care, ethnic minority, care users, service user dissatisfaction. 


\section{What is known about this topic?}

- Dissatisfaction with social care services is often reported by BME groups.

- Surveys are sometimes challenged as potentially not accurately representing service users' perspectives.

- Dissatisfaction has remained persistent despite some efforts to address this, suggesting that underlying issues have not been fully understood.

\section{What this paper adds?}

- The dissatisfaction is both real and encompassing of Pakistani, Bangladeshi and white British service users.

- Some underlying reasons for this are widely shared, whilst others have elements specifically related to particular cultural factors.

- Collaborative approaches are important to identifying viable solutions to address entrenched issues. 


\section{Introduction and background}

All English Local Authorities, which hold responsibility for the provision of care in their administrative area, routinely collect data about user experiences of and satisfaction with adult social care services. Recent Adult Social Care surveys have shown that black and Minority Ethnic (BME) groups report lower levels of satisfaction with social care services than the white British group. For example, in 2012-13, 65\% of white respondents reported being extremely or very satisfied with the services received. In contrast, this level of satisfaction was reported by $54 \%$ of Asian and Asian British service users (primarily those of Bangladeshi, Indian, Pakistani and Sri Lankan origin) and 55\% of black and black British service users (HSCIC, 2013). This is consistent with findings from previous years (NHS Information Centre, 2012). The survey also collects information on social care quality of life and the scores for BME groups are again consistently below those for the white British group (HSCIC 2013).

These survey findings are possibly explained by literature showing the impact of ethnicity on inequalities in provision and on experiences of services. Issues include persistently unresponsive (mental health) services (Bowl, 2007); staff who feel illequipped to address diversity (Richardson et al, 2006); services that fail to engage with BME groups (Cooper et al, 2010; Manthorpe et al, 2009); cultural assumptions made by service providers that prevent sensitive service provision (Chau and $\mathrm{Yu}$, 2009); and a continuing predominance of approaches which fail to appreciate intraethnic diversity (Williamson and Harrison, 2010) and the need to treat service users as individuals. While efforts have been made to provide care that is culturally appropriate, Croot (2012) found that the concept of 'cultural competence' (Yeowell, 2010) can be problematic if cultures are presented as monolithic, resulting in the inadvertent promotion of ethnic and cultural stereotypes. Jirwe et al. (2009) concluded that just knowing about cultural practices is not sufficient for the provision of responsive and needs based care.

Evidence on help-seeking behaviours shows poor uptake of health services among BME groups, either because people do not seek help or because services are not receptive to them (Hanley, 2007, Mir and Sheikh, 2010). Factors which affect service uptake include the normalisation of problems, so that help is not sought (Wells and Wagg, 2007) and the stigma attached to seeking help (Knifton, 2012 in the context of mental illness and Singh et al, 2012 in the context of diabetes). Users' experiences of services show similarities between groups, as well as differences. However, overall the literature on ethnicity and inequalities in provision points to a nuanced and variable interaction of cultural practices with other influences (socioeconomic, family and individual health and wellbeing) (Parveen et al, 2011).

In response, traditional models of provision, such as the generic 'one size fits all' and the 'culturally specific' frameworks, have given way to a more individualised approach to care provision which gives people the opportunity to specify the services they actually need (Gask et al, 2011). Some authors advocate a co-production model of care delivered by 'reflective practitioners' who take into account individual values, expectations and cultural practices (Almond and Lathlean, 2011; Atkin and Chattoo, 2007; Mir and Sheikh, 2010). 
Another explanation for the lower levels of satisfaction reported, however, is that satisfaction surveys themselves are culturally and linguistically inappropriate. Such surveys have not been validated specifically with people from BME communities and there is no evidence in the existing literature that survey questions are understood in the same way across ethnic groups. Language use and understanding of the concept of 'satisfaction', as formulated in social care survey questions, may not be consistent among groups of service users. This study explored this concept of satisfaction both in terms of cognitive understanding and lived experience.

This paper discusses the findings from a study which explored the reasons for consistently low levels of satisfaction with social care services amongst Bangladeshi and Pakistani service users in England in existing surveys (HSCIC, 2013; NHS Information Centre, 2012). The terms 'Bangladeshi' and 'Pakistani' refer to ethnicity, not nationality and include British Bangladeshis and British Pakistanis. The research used primary data collection using qualitative methods to explore both the realities behind responses to survey questions and the ways in which the issues could be addressed. A phenomenological approach was used to understand individual lived experiences, focussing on in-home adult social care provision arranged by the local authority. The study examined the question from two angles. First, an assessment of whether these surveys measure satisfaction in a consistent manner, to determine whether the differences between groups are genuine. Second, a detailed exploration of the experiences of accessing and using services. This paper focuses on the second angle. Subsequently, the research findings were used in collaborative workshops to develop recommendations to improve satisfaction that were grounded in real experiences of social care use.

\section{Study aims and objectives}

The research had three stages:

- an exploration of how service users and their families understand and respond to survey questions about satisfaction with social care (cognitive interviews);

- the collection of experiences of receiving care from the viewpoints of service users and their families and perspectives on providing care as expressed by social workers, provider agencies and care workers (this is the main focus of this paper);

- the validation of research findings and articulation of policy and practice recommendations through collaborative workshops in three English regions.

\section{Methods}

\section{Design}

The emphasis of the study was to elicit individual perspectives and interpretations. The investigation covered outward appearances (care provider-service user context and experience) and inward consciousness based on culture and values (expectations and interpretations of experiences of care, individual and structural contexts and personal values and practices linked to ethnic and faith backgrounds) (Creswell 1998). Methods for exploring the experience of service users drew on the principles of phenomenological inquiry (Creswell, 1998; Giorgi, 1985; Gubrium and Holstein, 1994; Moustakas, 1994) to gather and to understand "the meaning of 
several individuals of their lived experiences of a concept or phenomenon"(Creswell 1998 p.57).

To explore understanding of 'satisfaction' and language use as formulated in social care survey questions, 34 cognitive interviews with service users (individual or paired with a relative) were conducted. Cognitive interviewing is a methodological approach to evaluating the sources of response error in survey questionnaires by exploring how survey respondents understand questions, recall information and provide an answer (Collins 2016, Willis 1999). Detailed notes of participants' meanings and interpretations of the wording of these survey questions were analysed (Gray et al, 2014). The rest of the methods section describes the other elements of the research (63 in-depth interviews and the research with providers) since this is the focus of the paper.

\section{The study samples}

A purposive sampling approach was used in the selection of areas to locate the study and to identify research participants.

\section{Study locations}

In England social care is delivered by local government administrations (Local Authorities), therefore the primary selection criterion was councils with adult social services responsibilities (CASSRs). An additional selection criterion was the size of the Pakistani and Bangladeshi population, resulting in a focus on urban areas with larger Bangladeshi and Pakistani communities. To capture variation in social care provision and local socio-economic profiles, the research was located in 3 cities (4 Local Authorities): Birmingham, Leeds and London (predominantly in Newham and Redbridge, two of Greater London's 33 local authorities). To strengthen the validity of the research findings, both stages of the research were carried out in each area and the analysis triangulated individual experiences and perspectives within local service delivery contexts.

\section{Service user sample}

The study focused on two BME groups: Bangladeshi and Pakistani service users. These groups were selected because 'Asian' and 'Asian British' service user categories showed lower levels of satisfaction and poorer social care outcome scores than any other BME group. The term 'Asian' encompasses very diverse populations and two groups were selected to understand and compare experiences of social care in detail. Previous health research has shown that health inequalities are more marked for the Bangladeshi and Pakistani sub-groups within the 'Asian' classification (Sproston and Mindell eds, 2006). Although culturally and linguistically distinct, these two group share a common religion (Islam) and both experience language and communication issues (Mir and Sheikh, 2010; Merrell et al, 2006). To better understand the factors influencing satisfaction with social care and to disentangle any cultural or linguistic issues, a comparator group of white British service users was included.

\section{Inclusion criteria}

Bangladeshi, Pakistani, and white British service users aged 18 were eligible to take part if they used one or more of the services set out in Table 1. Where the individual 
was not able to take part by themselves due to language issues, illness or disability, a family member could take part in an interview instead of or together with the service user.

A short screening questionnaire was used to check for eligibility and recruitment was supported by community groups, social care providers and a specialist recruitment agency. In total, 63 service user interviews were conducted. Table 2 lists the characteristics of participants.

\section{Service provider sample}

Across the three areas, 24 staff involved in managing or providing care at public and private social care providers were interviewed (see Table 3). All of them had direct experience of providing care to adults of black and Asian ethnic minority groups.

\section{Data collection}

Interview topic guides for service users and social care providers were developed using themes identified from the research literature.

Interviews with service users and their families lasted around 60 minutes and were conducted by trained qualitative research specialists. Researchers attended fieldwork briefings where the study context, the methods and topic coverage were discussed. These covered daily routines, formal care, and informal care. An exploration of cultural and personal expectations, and experiences of accessing and receiving social care sought to identify drivers of satisfaction and dissatisfaction.

Focus groups were conducted with social workers and home care workers and indepth interviews with social care managers. Topics mirrored those used for the service users, and perspectives and experiences of providing care were explored.

\section{Data analysis and development of recommendations}

Data collection encounters were digitally recorded and transcribed. Interviews in community languages were translated and transcribed by the interviewers (Urdu and Punjabi) or by a professional translation agency (Bengali and Sylheti). Verbatim transcripts were charted ${ }^{2}$ using Framework in Nvivo10 (Ritchie and Spencer, 2003). A phenomenological approach to data analysis was undertaken, involving the reduction of data into relevant themes which were compared and contrasted. The coded data was interrogated with a view to maintaining a balance between the subjective and objective (Moustakas, 1994). This meant that while the validity of the accounts of lived experiences of social care users were paramount and held subjective value; these were compared and contrasted with the accounts of service providers in order to understand "the prejudices, viewpoints or assumptions regarding the phenomenon under investigation" (Katz, 1987 as cited in Patton, 2002). These subjective experiences were contextualised within the more objective structural construct of the social care system. This resulted in thick description and thematic synthesis which supported the formulation of recommendations. A reflexive approach during the data analysis process helped researchers to maintain distance from the data.

\footnotetext{
2 Charting refers to the process of managing and analysing qualitative data using Framework - a particular approach developed by Ritchie and Spencer (2003).
} 
An initial analytical stage was followed by three collaborative workshops (one in each area) with social care managers and practitioners, and service users. The half day workshops included plenary sessions and break-out group discussions during which researchers worked with participants to articulate recommendations that could improve satisfaction levels. This stage of the research was instrumental in validating the research findings and for setting out relevant and implementable recommendations.

\section{Ethics and consent procedures}

Ethical approval was obtained from the Social Care Research Ethics Committee supported by the Social Care Institute for Excellence (scie.org.uk).

Information about the research was available in accessible formats and translated into the relevant written languages (Urdu and Bengali). The research was also explained verbally and participant consent was sought at the start of each data encounter. The Interviews were offered in the main languages spoken by study participants (Urdu, Punjabi, Bengali and Sylheti). Service users were offered $£ 20$ to thank them for their participation.

\section{Results}

\section{Cognitive understanding of survey questions}

We found almost no evidence of ethnic differences in how Bangladeshi and Pakistani service users understand and respond to the survey questions compared with white British service users. Understanding and interpretation of satisfaction, quality of life, dimensions such as food and drink, and safety were consistent even when interpreted in a way which made them relevant to the service user. We found that unsuitable translations, in Urdu in particular, resulted in difficulties with reading the script and understanding particular words. However, the percentage of respondents using the translated questionnaire was very low (only three of the 2,410 Asian respondents in 2011/12 (NHS Information Centre 2012, Gill et al, 2014). It was more common for this group to have the English questionnaire translated by someone helping them with the questionnaire (36\% in 2011-12: NHS Information Centre, 2012). Evidence from the cognitive interviews showed that inconsistencies in understanding survey questions do not explain ethnic differences in satisfaction in the survey data. Our results suggest that differences in satisfaction between groups reflect real differences in satisfaction rather than inconsistencies in data collection. The cognitive findings are reported elsewhere (Gray et al, 2014).

\section{Care pathways common to all groups}

Analysis of social care pathways identified three aspects that played an important role in perceived satisfaction with care: accessing care through the local authority; interaction and communications with social workers; and receiving care from care and support workers. It was clear that there were common drivers of satisfaction for all three ethnic groups, as we now describe.

\section{Accessing care}

Ease of making contact with social workers, the speed at which an assessment was provided, and the care package agreed underpinned user satisfaction for all three ethnic groups. 
'Those that don't ask don't get anything'

(Female service user, Pakistani origin, Leeds)

However, requests for care did not always translate into the desired care package.

Aside from services to address unmet needs (such as loneliness and isolation), more equipment and more time from care and support workers, in particular, were identified as care gaps.

Communication with social workers

Reliability and consistency emerged as two main concerns in relation to communication. Reliability related to social workers keeping appointments and being responsive to users' need for contact. A common complaint was that it was difficult to make contact with social workers generally, and not having an assigned social worker hindered communication.

Frequent changes in social workers combined with perceived inadequate handovers meant users had to explain their situation and care needs repeatedly. Those who relied on social workers' knowledge to navigate the care system felt dissatisfied with what was viewed as sporadic communication.

\section{'We don't have any specific social worker at the moment. We have to contact the social services team in the area to get hold of the actual social worker.' \\ (Relative of male service user, Bangladeshi origin, Birmingham)}

\section{Receiving care}

The most significant driver of satisfaction was the nature of care received. Alongside task oriented care, there was an expectation of 'caring behaviour', based on service users' assessment of the care worker's attitude and approach to carrying out tasks. Therefore, the manner in which care was delivered mattered almost as much as whether the required tasks were completed. Satisfaction with care was expressed when the care worker was perceived to 'go above and beyond' the specific delivery of care tasks. This was particularly salient for service users experiencing loneliness and isolation.

Time pressures on those providing care due to inadequate time allocation to perform tasks and to travel between locations resulted in rushed or late appointments and made it difficult to 'go above and beyond' the required care. This time pressure was a source of dissatisfaction for service users and carers alike:

'...One is punctuality, two is the rapport and three is getting the work done properly. She's [my care worker] got all three. If you haven't got all three, then it might be a problem' (Service user, male, white British, London) 


\section{Satisfaction, ethnicity and culture}

Although there were common factors which contributed to satisfaction or dissatisfaction among all groups, the research found that there were cultural and ethnic differences which affected experiences of social care. Cultural issues were interpreted and expressed differently by service users, by their families (including carers), and by local authorities or providers.

\section{They 'take care of their own'}

There was a perception among local authorities, borne out in practice that Bangladeshis and Pakistanis with care needs were more likely than other groups to live with their families. This led to an assumption of a preference for 'taking care of their own'. This perception worked in parallel with an expectation within these groups that the family should or would provide care, resistance to 'outsiders' providing care in the home and a perceived stigma associated with seeking care outside the family. This perceived and internalised obligation to provide care meant that these groups were less likely than their white British counterparts to be aware of their entitlements to services:

\section{I think within the Asian community, sometimes they don't ask for that extra help, because it's very much not seen as the done thing, really ... And the ones that then do have a carer coming in ... families have said they almost feel as though they're being watched, or they're almost labelled.' (Senior manager, day opportunities provider)}

In instances where a number of these factors intersected, Bangladeshi and Pakistani people were less likely to seek assistance, resulting in long-term unmet need and with services being sought only at a crisis point. Dissatisfaction was caused when services were slow to respond; at the same time there was satisfaction with the care finally received after long periods of coping without services.

\section{Family and caring}

The research found evidence of misunderstandings about the role of care providers among family members, which was, in some cases, associated with an expectation of meeting the need of wider family members as well as the service user. This could result in dissatisfaction as such expectations were unlikely to be met. Furthermore, differing religious or cultural preferences among family members and service users could result in dissatisfaction as care providers could not meet the different preferences of everyone involved. The experiences of carers were not explored in detail in this project, however it was clear that carers appreciated the support that they received, and that service providers and local authorities were concerned about the experiences of carers:

'It would be hard [without formal care services], it would be hard. It's made it a little bit easier, I mean I can leave [service user] with them and go out shopping or to somebody's house or go to town. Or they can take her out while I can have a bit of break. Although l've been looking after her, I did courses during the day because if I'd stayed inside then I would have got more depressed.'

(Relative of Female Service user, Pakistani origin, Leeds) 


\section{Gendered caring}

In many cases the majority of care was provided by family members and supplemented by a small amount of formal care from the local authority. Across all ethnic groups women were more likely than men to be providing additional care. The pattern of mothers caring for adult children with disabilities, women caring for husbands, and daughters and daughters-in-law caring for parents was stronger among Bangladeshi and Pakistanis than among the white British group.

The intersection of female dominated caring and the assumption on the part of some care providers that Asian families preferred to 'care for their own', combined with economic activity or looking after young children placed a heavy caring burden on women. Where the caring responsibility was combined with poor literacy and English language skills, accessing formal care became even more difficult. In these cases, access to care was mediated by those who had better English skills, mainly men.

The inability to communicate with the main carer was of concern to providers and local authorities who relied on other family members for interpretation.

'We find that sometimes when we go to the assessment it's the son that's sitting there, but he's not the one that's providing the care - it's his wife. ... And you have to keep saying to him, "Can I speak to your wife?" And he'd be going, "Well, she can't speak English." (Social worker)

Disentangling reasons for dissatisfaction is difficult where the wider family is involved - care users, their main carers and those who act as communicators with the care system. It is possible that the extent of dissatisfaction may even be underestimated in these situations.

\section{Language and communication barriers}

Communication barriers were experienced along the care pathway. Accessing care, especially through local authority automated phone lines was particularly difficult for Bangladeshi and Pakistani people. Where language services (interpreter or own language staff) were not available, difficulties were encountered in communications with social workers; in challenging care packages; explaining tasks, and building rapport with care workers.

Factors that mediated the negative consequences of poor communication were levels of education; English language proficiency; the involvement of family members; and the assistance of third parties such as community organisations or providers who advocated on behalf of carers. A shared language increased satisfaction with care workers, however this was not essential and the use of body language and signing helped to build rapport between care workers and service users, contributing to higher levels of satisfaction with care:

'If I don't understand anything, they [care workers] call my daughter so that she can make me understand.' (Relative of female service user, Bangladeshi origin, Leeds)

Ethnic matching as a response to cultural and religious difference 
For the most part, an accepted response to cultural diversity (by both providers and service users) was the matching of care providers to the cultural characteristics of service users.

A common language; appropriate and respectful forms of address (for example, addressing older care users as 'aunty' instead of using first names); preparation of culturally appropriate food; the gender of care workers; religion (for support with ritual ablution for prayer); and a general cultural understanding to help build rapport and familiarity were identified by service users as important dimensions of ethnic matching. The salience and relative value of these dimensions was specific to individual service users. For those concerned about preserving the privacy of family discussions, religion matching was more important than language matching. The use of personal budgets to employ family members as carers could facilitate the provision of culturally appropriate care:

\section{'I think it's very important to understand, to have the same background... it's only because of the food, especially if my daughter go[es] out, I need to make sure whoever is supporting [ her will] make sure that she eat[s] halal food...culture and religion is very important, and other people [carers] should respect that.' (Relative of a female service user, Pakistani origin, London).}

Even if some type of ethnic matching was preferred, white British care workers were perceived to perform practical tasks such as cleaning and changing clothes more effectively than care workers of other ethnicities. Importantly, care user-care provider ethnic matching was not always necessary to improve satisfaction.

\section{Meeting service users' needs}

The criteria underpinning ethnic matching carried out by providers was questioned by service users and their families as in some cases it resulted in inappropriate or inadequate matching. However, from the provider and local authority perspectives ethnic matching based on requested dimensions was not always possible so compromises had to be made, for example matching South Asian origin but not language. Importantly, meeting cultural needs was not just about matching care workers but could include appropriate matching of resources (such as installation of a bathroom that facilitates ritual ablution before prayer).

There was no evidence of cultural awareness training ${ }^{3}$ for care workers. Despite this, there were care users and relatives who felt that the provision of care was underpinned by some level of cultural awareness and this contributed to satisfaction. Even small gestures of cultural understanding, such as using appropriate and familiar forms of address, could have a positive effect.

The contrasting view was that cultural familiarity could undermine the fundamentally professional (and formal) nature of the care user-care worker relationship. Poor understanding by service users and poor communication by service providers of the

\footnotetext{
3 In England evidence informed guidelines on cultural awareness in social work (for example, http://www.etn.leeds.ac.uk/document/resources/RPFA_Outline_14.pdf) as well as training programmes such as that offered by the social care training hub (http://thesocialcaretraininghub.co.uk/en/f2f-course/cultural-awareness) are available.
} 
balance between cultural appropriateness and a formal care giving relationship hindered care provision that met care users' needs and expectations:

'Work with the service user and listen to their needs. A particular service user will say, "I want someone from my community because she speaks my language. I like it - somebody comes in, in the day and I can speak my language." Or, "I confide in her." Or, "It's like a daughter coming to the door." Whereas another service user will turn round and say, "It's my private life. I don't want somebody to come in and intrude and ask me all questions about - where's your daughter? I don't want any of that so send me a care worker that's not from the community." So ... you're listening to the service user and what they need, on an individual basis, and not generalising.' (Senior manager, homecare provider)

\section{Addressing the issues}

The collaborative workshops generated several suggestions for practical ways to embed culture awareness within person-centred care packages. These included:

- Raising awareness of available services through local media and community organisations in order to faciliate access to services and with a view to reducing the stigma associated with accessing services;

- Effective communication between local authorities and social care providers of service user characteristics, preferences, and cultural and religious needs so that these can underpin the development of an appropriate person-centred package of care;

- Implementing a service user file, 'Ten things you need to know about me', to be updated by service users' as needed and with the support of care workers.

- Support packages that take into account the support needs of family members (usually women) providing informal care;

- Effective modes of communication for those who are not fluent in English and greater use of better trained and well briefed interpreters with workers who know how to work with an interpreter, as well as more face-to-face contact between provider and service user.

- Recruitment of a local care workforce that represents the diversity of the service user population and effective promotion of the profession within BME populations.

- Development and roll-out of a training programme in cultural awareness and its application to social care practice for social workers and care workers this would cover specific knowledge as well as guidance on how to ask service users about what kind of support would be appropriate.

\section{Discussion and conclusions}

Our findings confirm the continuing reality of service user dissatisfaction highlighted in earlier studies (Manthorpe et al, 2009; Griffith, 2008; Astin et al, 2008; Merrrell et al, 2006). Whilst, as we have shown, dissatisfaction extends across all three groups of service users, there are particular factors that come into play for those of minority ethnicity.

The research findings add more detailed knowledge to the known range of barriers that hinder the provision of culturally appropriate care. For example, ethnic matching 
is not a simple process; comprising a number of dimensions, each of which has varying salience according to individual preferences and beliefs. In addition, the expectations of the care user or their family may result in misunderstandings about what care can be provided and dissatisfaction where those expectations are considered unrealistic by care providers. The research also offers a range of practical recommendations for improving service users' satisfaction with care.

Our findings confirm that a person-centred approach to meeting the needs of diverse clients is needed irrespective of service user's ethnicity or cultural background. (Bowes et al, 2012). Results suggest that the social and cultural background of individuals working in social care can lead to assumptions being made about what service users need and want. This implies that local authorities, providers and individual care workers need to be aware of their institutional and individual cultural perspective and of the predominant values ingrained in professional practice (Salway et al, 2013). At the same time cultural awareness of the local communities in which care is provided has to be developed. In the case of Bangladeshi and Pakistani service users, a lack of cultural awareness can result in inappropriate care or care provision that is based on cultural and religious stereotypes. Our findings suggest that services should explore BME carers' needs in order to provide more culturally appropriate responses. This would build a more nuanced understanding of individuals' cultural context so that socially acceptable approaches can be developed to address underuse of services by BME communities. Such coproduced solutions are more likely to help reduce the stigma of seeking help than those that require individuals to fit into existing systems of care (Almond and Lathlean, 2011; Knifton, 2012; Singh et al, 2012). The needs of carers were not fully explored in this research and would be a fruitful avenue for further work.

Evidence from this study suggests that higher levels of service user satisfaction are likely to be achieved by a person-centred approach and better communication, between different levels of care provision (e.g. local authorities and provider organisations) and also between care workers and service users. Sharing of information on ethnicity and cultural needs between local authorities and providers, for example, would prevent service users having to repeat their needs to different professionals. This level of knowledge sharing could facilitate the provision of care to meet individual need in a timely way. Clear communication about what services can and cannot provide would also help with managing service users' expectations of care while providing essential information to people receiving services and their families.

Together, the analysis of the data collected and the ideas generated from the collaborative workshops imply that how care workers take their normative, standard practice and combine it in meaningful ways with salient cultural and religious dimensions of care requires a type of 'cultural shift' in practice which involves both structures and individuals. Effective communication stands out as a key challenge, whether this is systemic (between for example, local authorities and care providers) or relational, most importantly between care workers and service users. A further challenge is to develop an understanding of the care system among service users in order to facilitate access to care and decrease the gatekeeping role of professionals. Alongside this, care workers and their employers need to invest in developing knowledge and awareness of relevant cultures and religions in order to embed this in 
their daily practice. Some of this may come from training programmes, but allowing care workers the time and space to learn from the people for whom they care is also likely to be a worthwhile investment for social care commissioners.

\section{Strengths and Limitations}

An inclusive and flexible approach was undertaken to conduct interviews in different languages and with service users alone or in paired interviews with carers. Where possible, ethnic and gender matching of interviewer-interviewee was implemented. The research was strengthened by this flexible approach as well as by the inclusion the perspectives of social care providers. The collaborative workshops to develop recommendations were a unique aspect of the study.

Recruitment of service users and providers was challenging despite the use of different strategies. Organisations were under resourced and sceptical about the research and therefore unwilling to help with recruitment or to take part. This was a small exploratory study and it is important that this is built on by ensuring the recommendations are put into practice and conducting further research. 


\section{References}

Almond, P., and Lathlean, J. (2011). Inequity in provision of and access to health visiting postnatal depression services. Journal of Advanced Nursing, 67(11), 23502362.

Astin, F. F., Atkin, K. K., and Darr, A. A. (2008). Family support and cardiac rehabilitation: A comparative study of the experiences of south Asian and whiteEuropean patients and their carer's living in the United Kingdom. European Journal of Cardiovascular Nursing: Journal of the Working Group on Cardiovascular Nursing of the European Society of Cardiology, 7(1), 43-51.

Atkin, K., and Chattoo, S. (2007). The dilemmas of providing welfare in an ethnically diverse state: Seeking reconciliation in the role of a 'reflexive practitioner'. Policy and Politics, 35(3), 377-393.

Bowes, A., Dawson, A. and Greasley-Adams, C. (2012) Satisfaction with adult social care among Bangladeshi, Pakistani and white British populations: Literature Review. http://www.natcen.ac.uk/media/563050/bme-social-care-satisfaction-literaturereview-2014.pdf (accessed 22/11/2015)

Bowl, R. (2007). The Need for Change in UK Mental Health Services: South Asian Service Users' Views. Ethnicity and Health, 12(1), 1-19.

Chau, R.C.M., and Yu, S.W.K. (2009). Culturally sensitive approaches to health and social care. Uniformity and diversity in the Chinese community in the UK. International social work, 52(6), 773.

Collins, D (2016) Cognitive Interviewing Practice. London: Sage

Cooper, C., Tandy, A.R., Balamurali, T.B.S., and Livingston, G. (2010). A Systematic Review and Meta-Analysis of Ethnic Differences in Use of Dementia Treatment, Care, and Research. American journal of geriatric psychiatry, 18(3),193-203.

Creswell, A. (1998) Qualitative Inquiry and Research Design: Choosing Among Five Approaches. Thousand Oaks: Sage.

Croot, E. J. (2012) The care needs of Pakistani families caring for disabled children: how relevant is cultural competence Physiotherapy 98,4:351-356

Gask, L., Aseem, S., Waquas, A., and Waheed, W. (2011). Isolation, feeling 'stuck' and loss of control: Understanding persistence of depression in British Pakistani women. Journal of Affective Disorders, 128(1-2), 49-55.

Gill, V., Husain, F., Vowden, K., Aznar, C., Blake, M. (2014) Satisfaction with social care services among black and Minority Ethnic populations: Exploring satisfaction with adult social care services amongst Pakistani, Bangladeshi and white British 
people. NatCen Social Research. http://www.natcen.ac.uk/media/563048/bmesocial-care-satisfaction-main-report-2014.pdf (accessed 22/11/2015)

Giorgi, A. (1985) Phenomenology and Psychological Research. Pittsburgh, PA: Duquesne University Press

Gray, M. Lepps, H. and Blake, M. (2014) Exploring satisfaction with social care services amongst Pakistani, Bangladeshi and white British populations: Findings from cognitive interviews. NatCen Social Research.

http://www.natcen.ac.uk/media/563051/bme-social-care-satisfaction-cognitive-report2014.pdf (accessed 22/11/2015)

Griffith, L. (2008) Complaints, sensitivities and responsibility: An ethnographic investigation into the debates concerning the care of Bangladeshi mothers in the East End. International Migration, 46(5), 143-166.

Gubrium, J.F.and and Holstein, J.A. (1994) 'Analyzing talk and interaction', in J.F. Gubrium and A. Sankar (eds), Qualitative Methods in Aging Research. Newbury Park, CA: Sage. pp. 173-88.

HSCIC (2013) Personal Social Services Adult Social Care Survey, England 2012-13, Adult Social Care statistics team, Health and Social Care Information Centre (Final Release: December 2013).

Hanley, J. (2007). The emotional wellbeing of Bangladeshi mothers during the postnatal period. Community Practitioner: The Journal of the Community Practitioners' and Health Visitors' Association, 80(5), 34-37.

Hunt, S. H., andand Bhopal. R. (2004). Self report in clinical and epidemiological studies with non-English speakers: the challenge of language and culture. Journal of Epidemiology and Community Health, 58, 618-622.

Jirwe, M., Gerrish, K., Keeney, S., and Emami, A. (2009). Identifying the core components of cultural competence: findings from a Delphi study. Journal of Clinical Nursing 18,18:2622-2634.

Katz, L. (1987). The experience of personal change. Unpublished doctoral dissertation, Graduate College, The Union Institute, Cincinnati, $\mathrm{OH}$.

Knifton, L. (2012) Understanding and addressing the stigma of mental illness with ethnic minority communities Health Sociology Review, 21,3:287-298

Manthorpe, J., lliffe, S., Moriarty, J., Cornes, M., Clough, R., Bright, L. andand Rapaport, J. (2009). 'We are not blaming anyone, but if we don't know about amenities, we cannot seek them out': black and minority older people's views on the quality of local health and personal social services in England. Ageing and society, 29(1), 93-113.

Merrell, J., Kinsella, F., Murphy, F., Philpin, S. and Ali, A. (2006) 'Accessibility and equity of health and social care services: exploring the views and experiences of 
Bangladeshi carers in South Wales, UK' Health and Social Care in the Community 14(3), 197-205.

Mir, G., and Sheikh, A. (2010). 'Fasting and prayer don't concern the doctors ... they don't even know what it is': Communication, decision-making and perceived social relations of Pakistani Muslim patients with long-term illnesses. Ethnicity and Health, 15(4), 327-342.

Moustakas, C. (1994) Phenomenological Research Methods. Thousand Oaks: Sage.

Parveen, S., Morrison, V., and Robinson, C. A. (2011). Ethnic variations in the caregiver role: A qualitative study. Journal of Health Psychology, 16(6), 862-872.

Patton, M. Q. (2002) Qualitative Research and Evaluation Methods: Integrating Theory and Practice. Thousand Oaks: Sage.

Richardson, A., Thomas, V. N. and Richardson, A. A. F., (2006). 'Reduced to nods and smiles': Experiences of professionals caring for people with cancer from black and ethnic minority groups. European Journal of Oncology Nursing, 10(2), 93-101.

Richie, J. and Lewis, J (2003) Qualitative Research Practice. London: Sage.

Salway, S., Turner, D., Mir, G., Bostan, B., Carter. L., Skinner, J., et al. (2013) Towards equitable commissioning for our multiethnic society. Health Service Delivery Research 2013;1(14)

Singh, H., Cinnirella, M., and Bradley, C. (2012) Support systems for and barriers to diabetes management in South Asians and Whites in the UK: qualitative study of patients' perspective BMJ OPEN 2,6 e001459

Sproston, K. and Mindell, J. (eds). (2006) The Health Survey for England: The health of ethnic minority groups. Stationary Office.

The NHS Information Centre, Social Care Team (2012) Personal Social Services Adult Social Care Survey, England 2011-12 (Final Release). Health and Social Care Information Centre.

Wells, M. M., and Wagg, A. A. (2007). Integrated continence services and the female Bangladeshi population. British Journal of Nursing (Mark Allen Publishing), 16(9), 516-519.

Williamson, M. and Harrison, L. (2010). Providing culturally appropriate care: A literature review. International journal of nursing studies. 47(6), 761-769.

Willis, G. (1999) Cognitive Interviewing: A "How To" guide. The Research Triangle Institute. http://www.hkr.se/pagefiles/35002/gordonwillis.pdf

Yeowell, G. (2010). What are the perceived needs of Pakistani women in the north west of England in relation to physiotherapy, and to what extent do they feel their needs are being met? Physiotherapy, 96(3), 257-263. 


\section{Tables}

Table 1: Inclusion criteria for interview participants

1. Receiving care in the home (and not living in residential care settings)

2. Allocated a personal budget (direct payment) or care arrangements made by the Local Authority

3. Using day opportunity services (such as, luncheon clubs, exercise classes)

4. Receiving prepared meals (Meals on Wheels)

5. Having assistive equipment installed in the home

Table 2: Service user participants for in-depth interviews

\begin{tabular}{|l|l|}
$\begin{array}{l}\text { Sample } \\
\text { characteristics }\end{array}$ & $\begin{array}{l}\text { Number of } \\
\text { participants }\end{array}$ \\
\hline Gender & 28 \\
\hline Male & 35 \\
\hline Female & 30 \\
\hline Age & 33 \\
\hline $18-59$ & 20 \\
\hline $60+$ & 24 \\
\hline Ethnicity & 19 \\
\hline Bangladeshi & \\
\hline Pakistani & 13 \\
\hline White British & 12 \\
\hline Region & 38 \\
\hline Birmingham & \\
\hline Leeds & \\
\hline London & \\
\hline
\end{tabular}

Table 3: Social care provider participants

\begin{tabular}{|l|c|}
\hline \multicolumn{1}{|c|}{ Sample characteristics } & Number of participants \\
\hline LA senior manager & 2 \\
\hline LA Human Resources Manager & 1 \\
\hline Social Worker & 6 \\
\hline Day centre manager & 2 \\
\hline Home Care Manager & 2 \\
\hline Care workers & 11 \\
\hline
\end{tabular}


Table 4 Participant recruitment - organisations and individuals contacted

\begin{tabular}{|c|c|c|c|c|c|}
\hline \multirow[b]{2}{*}{ Area } & \multicolumn{2}{|c|}{ Number of organisations } & \multicolumn{3}{|c|}{ Number of individuals contacted } \\
\hline & $\begin{array}{l}\text { Service } \\
\text { users }\end{array}$ & $\begin{array}{l}\text { Social care } \\
\text { providers }\end{array}$ & $\begin{array}{l}\text { Local } \\
\text { Authority } \\
\text { managers }\end{array}$ & $\begin{array}{c}\text { Human } \\
\text { Resources } \\
\text { professionals }\end{array}$ & $\begin{array}{c}\text { Social } \\
\text { workers }\end{array}$ \\
\hline Birmingham & 45 & 15 & & & \\
\hline Leeds & 63 & 2 & & & \\
\hline London - Newham & 52 & & & & \\
\hline $\begin{array}{l}\text { London - } \\
\text { Redbridge }\end{array}$ & 33 & & & & \\
\hline $\begin{array}{l}\text { Other London } \\
\text { areas }\end{array}$ & 6 & 12 & & & \\
\hline Total & 199 & 29 & 8 & 2 & 8 \\
\hline
\end{tabular}

